\title{
A multiple objective mixed integer linear programming model for power generation expansion planning
}

\author{
C. Henggeler Antunes ${ }^{\mathrm{a}, \mathrm{b}, \mathrm{c}, *}$, A. Gomes Martins ${ }^{\mathrm{a}, \mathrm{b}, \mathrm{c}}$, Isabel Sofia Brito ${ }^{\mathrm{b}}$ \\ a Departamento de Engenharia Electrotécnica, Universidade de Coimbra, Polo II, 3030 Coimbra, Portugal \\ ${ }^{\mathrm{b}}$ Escola Superior de Tecnologia e Gestão, Instituto Politécnico de Beja, 7800 Beja, Portugal \\ c INESC-Coimbra, Rua Antero de Quental, 199, 3000 Coimbra, Portugal
}

Received 26 March 2001

\begin{abstract}
Power generation expansion planning inherently involves multiple, conflicting and incommensurate objectives. Therefore, mathematical models become more realistic if distinct evaluation aspects, such as cost and environmental concerns, are explicitly considered as objective functions rather than being encompassed by a single economic indicator. With the aid of multiple objective models, decision makers may grasp the conflicting nature and the trade-offs among the different objectives in order to select satisfactory compromise solutions. This paper presents a multiple objective mixed integer linear programming model for power generation expansion planning that allows the consideration of modular expansion capacity values of supply-side options. This characteristic of the model avoids the well-known problem associated with continuous capacity values that usually have to be discretized in a post-processing phase without feedback on the nature and importance of the changes in the attributes of the obtained solutions. Demand-side management (DSM) is also considered an option in the planning process, assuming there is a sufficiently large portion of the market under franchise conditions. As DSM full costs are accounted in the model, including lost revenues, it is possible to perform an evaluation of the rate impact in order to further inform the decision process.
\end{abstract}

(C) 2003 Elsevier Ltd. All rights reserved.

\section{Introduction}

Power generation expansion planning inherently involves multiple, conflicting and incommensurate objectives. Therefore, mathematical models become more realistic if distinct evaluation

\footnotetext{
* Corresponding author. Fax: +351-239-824692.

E-mail address: cantunes@inescc.pt (C.H. Antunes).
} 
aspects, such as cost and environmental concerns, are explicitly considered by giving them an explicit role as objective functions rather than aggregating them in a single economic indicator objective function. Multiple objective models can provide decision support to decision makers (DMs) by rationalizing the comparison among different alternative solutions, thus enabling the DM to grasp the inherent conflicts and trade-offs among the distinct objectives for selecting a satisfactory compromise solution from the set of nondominated solutions. In multiple objective models, the concept of optimal solution in single objective problems (unique, in general) gives place to the concept of nondominated solutions (feasible solutions for which no improvement in any objective function is possible without sacrifice at least one of the other objective functions).

The perspective of integrated resource planning (IRP) remains in the proposed model as one of the demand-side management (DSM) objectives that is most useful to utilities - peak clipping has been considered as a demand-side option in the planning process. This requires that the electricity market in the target region or country is organized in such a way that an important part of the load is supplied in a franchise environment and generation capacity expansion is mostly centrally planned. This still corresponds to a very large number of cases around the world, either because such electricity markets have not been liberalized or because the transformations kept some fundamental characteristics of the traditional market organization. The model takes into account the full cost of implementation and operation of the used DSM option, including lost revenues as an operational cost. Hence, the DM can perform an evaluation of the rate impact (if needed) that must be considered as an additional attribute of each solution chosen for consideration in the decision process. The model does not perform this evaluation but enables it to be carried out according to the procedures found most appropriate in each case.

Recently, multiple objective models have received wider attention in energy planning because of their capability for taking into account the multiple competing objectives that are generally pursued [5,8]. Power generation expansion problems have been modelled as multiple objective linear programmes $[3,6]$. However, the modular nature of the expansion capacities of generation groups cannot be appropriately tackled using continuous variables alone [7]. Some of those studies attempt to circumvent this intrinsic model shortcoming by using some form of approximation and/or discretization of the continuous solutions (to the multiple objective linear programming, MOLP) in order to comply with the available expansion modules.

This paper presents a new approach based on a multiple objective mixed integer linear programming (MOMILP) model to provide decision support in the selection of satisfactory compromise expansion plans. Some results are presented that have been obtained using an interactive procedure aimed at assisting DMs in the search process.

\section{Multiple objective (mixed) integer linear programming}

Nondominated solutions can be computed by optimizing a scalar function which is a nonnegative weighted-sum of the multiple objective functions [10]. The optimal solution to the weighted-sum scalarizing function, if unique, yields a nondominated vertex solution. However, by using this form of scalarization (i.e. transforming the multiple objective problem into the surrogate weighted-sum scalar problem), only nondominated vertex solutions can be computed. 
These solutions define the boundary of the convex hull of the nondominated solution set in the objective function space of the MOMILP problem.

Nondominated solutions located in the interior of the convex hull (i.e. those which are not vertices) cannot be reached using the weighted sum programme because they are dominated by a convex combination of vertex solutions, and hence cannot be optimal solutions to the weighted-sum function (no set of weights exists which define a supporting hyperplane for them). For this reason, though they are nondominated, they are generally called convex dominated solutions or unsupported (nondominated) solutions. Since these solutions (which are a likely occurrence) are actually nondominated, they must be considered as potential compromise solutions and consequently the algorithms must accommodate for their computation.

These concepts are illustrated in Fig. 1 for the two-objective case. Solutions 1 and 2 are the nondominated solutions which minimize $f_{1}$ and $f_{2}$ objective functions, respectively. Solutions 3 and 4 are other two vertex nondominated solutions. Solutions 5 and 6 are nondominated, even though they are dominated by a convex combination of vertex solutions 3 and 4 . The same happens with solution 7 with respect to solutions 2 and 4 . Solution 8 is dominated by solutions 4 and 7, and solution 9 is dominated by 3 . Zero is the ideal solution-the so-called ideal solution is the one that would optimize all the objective functions simultaneously, which is not feasible whenever the objective functions are conflicting.

However, the computational effort associated with the computation of the nondominated solutions to the MOMILP model is much higher than obtaining nondominated solutions for its MOLP counterpart (i.e. where the integer variables are relaxed to continuous ones).

The computational effort to determine all these solutions led to the use of an interactive approach to deal with the MOMILP model for power generation expansion planning. In interactive methods, decision phases alternate with computation phases. The DM intervenes in the solution search process by inputting information into the procedure, which in turn is used to guide the computation phase towards solutions which correspond more closely to his/her (evolutionary) preferences. An interactive method based on a reference direction algorithm (see Section 3) has been implemented to compute (supported and unsupported) nondominated solutions. At each iteration, the algorithm finds nondominated solutions to the relaxed

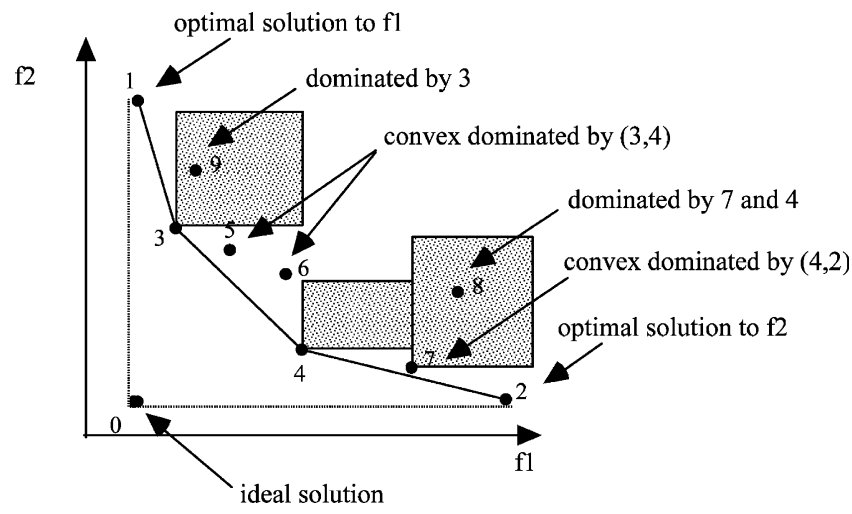

Fig. 1. Nondominated, convex dominated, dominated and ideal solution. 
MOMILP problem (i.e. to the MOLP), in order to reduce the computational burden. Only at certain iterations, if the DM identifies a relaxed nondominated solution as a good compromise, an additional MOMILP problem is solved to find a (supported or unsupported) nondominated solution by using the relaxed solution as the reference point. The reference point can also be adaptively formed by the DM by specifying the aspiration levels he/she would like to attain for each objective function (which act as the DM's guidelines in the interactive search process). The scalarizing process is based on computing the solution which minimizes the Tchebycheff $\left(\mathrm{L}_{\infty}\right.$ metric) distance to the reference point. In this way, both supported and unsupported nondominated solutions can be reached.

\section{An interactive algorithm for MOMILP problems}

An interactive procedure has been developed which combines the reference direction approach proposed by Narula and Vassilev [9] with the STEM method [2]. Initially, the nondominated solutions which are the individual optima to each objective function in the MOLP are computed. This is aimed at offering the DM a first overview of the ranges of each objective function over the nondominated region (to the MOLP). It also permits to identify the (continuous) ideal solution, which is initially used as a reference point (in the sense that it is the best the $\mathrm{DM}$ can achieve within the feasible region for each objective function individually). The MOLP problem is a relaxation of the MOMILP problem by ignoring the integer constraints on decision variables in order to reduce the computational burden.

The nondominated solution, to the MOLP, which minimizes the maximum distance (i.e. using a Tchebycheff metric) to the reference point is then computed. This metric captures the DM's attitude of taking into account the worst deviation to his/her aspiration levels (which define the reference point).

If the DM considers this (continuous) solution as a satisfactory one, then it is taken as the new reference point and the nondominated solution to the MOMILP which minimizes the Tchebycheff distance to it is computed. Otherwise, the DM is asked to express his/her preferences either by specifying a new reference point (aspiration levels he/she would like to attain for each objective function) or indicating the objective functions that already possess satisfactory values and which he/she is willing to relax (accept to degrade) by a certain amount in order to improve the other functions.

This latter form of preference expression implies introducing new "soft constraints" on the objective function values ("soft" meaning that they can be modified or removed at a later interaction with the DM) acting as reservation levels.

In case the DM finds the nondominated solution to the MOMILP satisfactory, then the interactive process stops, and this solution is the selected compromise solution. Otherwise, new DM's preferences, as described above, are required and a new nondominated solution to the MOLP is computed.

In this way, the method only resorts to the computation of a solution to the MOMILP, which imposes a heavier computational burden, whenever the DM recognizes a nondominated solution to the MOLP as an interesting one. 
Within this framework, the interactive process is understood as a learning process in which the DM can go through the nondominated region in a progressive and selective way by using the information gathered so far to express new preference information to guide the ongoing search for new solutions.

The block diagram of the proposed interactive approach to provide decision support in MOMILP problems is depicted in Fig. 2.

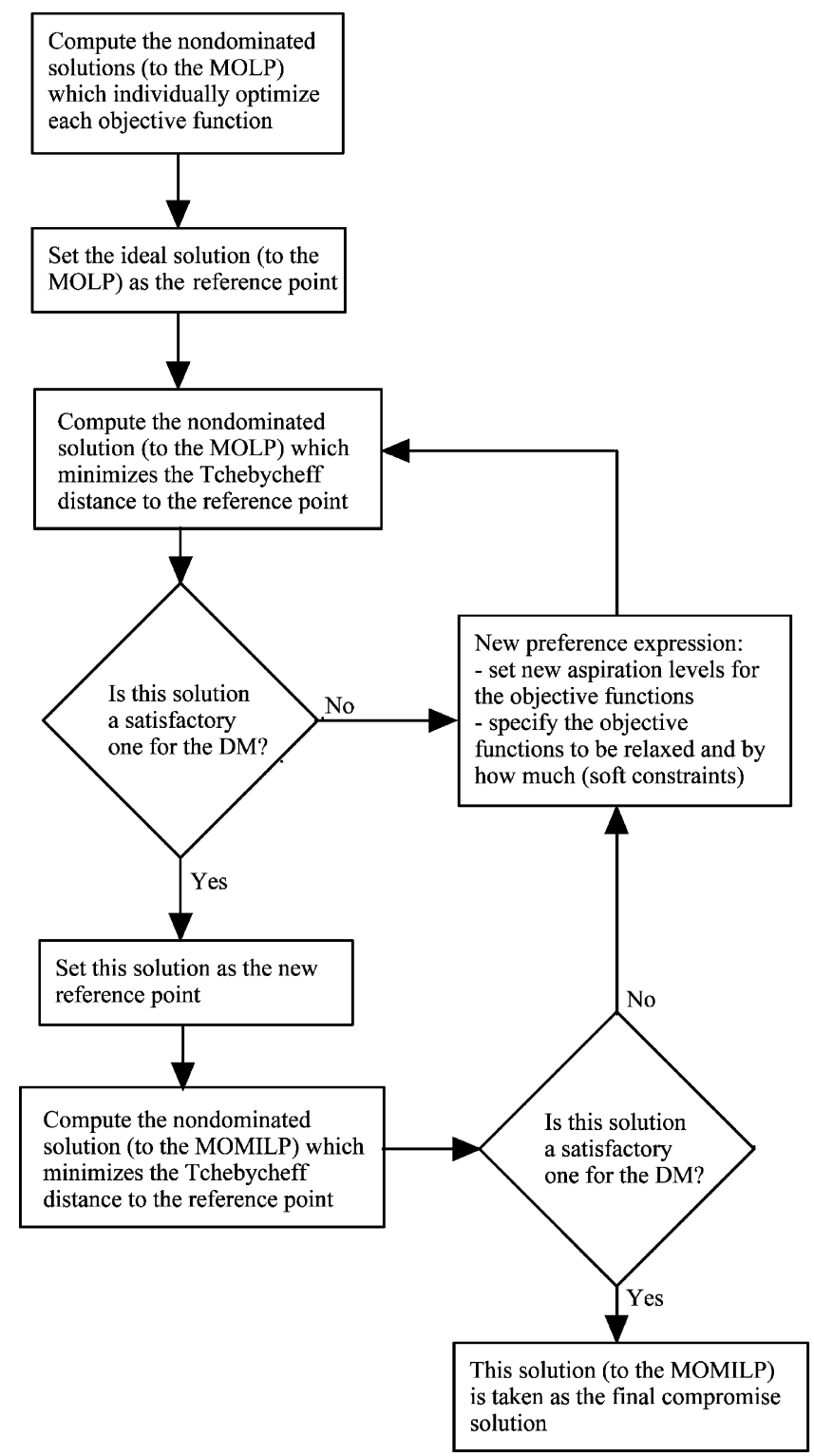

Fig. 2. Flowchart of the interactive procedure to deal with MOMILP problems. 


\section{Mathematical formulation of the MOMILP model}

The model considers three objective functions which quantify: the total expansion cost, the environmental impact associated with the installed power capacity and the environmental impact associated with the energy output. There are six categories of constraints related to: the reliability of the supply system, the availability of the generating units, the capacity of equivalent DSM generating group, the total capacity installed throughout the planning period, the pollutant emissions and the available capacity modules for expansion for each generating technology. Decision variables refer to the power to be installed and energy output of generating technologies considered for additions (gas, both simple and combined cycle, coal and DSM unit) and those existing at the beginning of the planning period (coal and oil).

The improved $z$-substitute method [1] has been used to model load demand. This method, already used in previous studies by the authors [3,6], enables a reduction in the number of constraints. In the improved $z$-substitute method, the planning period is divided into subperiods, each one corresponding to a load duration curve (LDC). Subperiods are composed of a specified number of intervals, each one corresponding to a power demand value. Decision variable $z_{i s}^{j}$ is the reduction in power output of groups of type $i$ occurring from interval $s-1$ to interval $s$ in subperiod $j$ (Fig. 3).

DSM actions focus on changing the pattern of demand for electricity, including generic load shape objectives such as peak clipping, valley filling, load shifting, flexible load shape, strategic load growth and strategic conservation [4]. In practice, the goal is generally to reduce peak electricity demand and DSM often refers to actions aimed at reducing demand and changing timeof-use patterns. Therefore, peak clipping has been selected to be incorporated into the model,

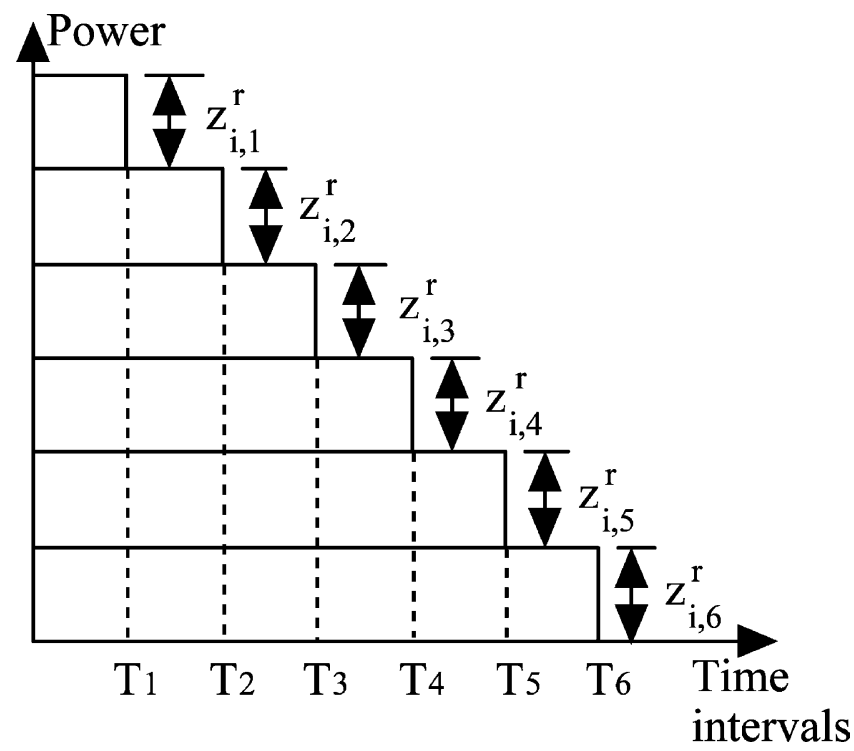

Fig. 3. Contribution of groups of type $i$ in subperiod $r$ to the corresponding LDC. 
because it may be implemented by means of procedures which are actually used by utilities, such as direct load control (DLC) and time-of-use rates.

DSM has been modelled as an equivalent DSM generating unit, which is used, from the point of view of satisfying the forecasted demand, to replace peaking units and contributes to the generation system reliability when available, even if not in effective action. The same type of parameters used to characterize the supply-side generating units are used to model the DSM unit: implementation costs (installation and removal costs, initial contacts with the customers and incentives paid at the beginning of the DSM programme), operation cost (equipment operation and maintenance charges, incentives paid on an annual basis and lost revenues) and environmental impact (included in the model for the sake of generality). In this model, the DSM unit assumes an actual meaning of avoided peak generation because it is allowed to be effective only for the highest demand values in each LDC.

Generating groups already existing at the beginning of the planning horizon are considered in the model along the ones available for capacity expansion. The following parameters are used to characterize the generating groups: primary energy source, installed capacity, availability factor (to account for maintenance effects on energy production), investment costs, operational and maintenance charges, environmental impacts with respect to nonmonetized effects, costs of environmental externalities and the discrete nature of actual capacity expansion modules. Reliability is modelled by defining a reserve margin for the whole electric power system, including the DSM options. Upper bounds have been imposed to the total capacity of each generating technology to be installed in each subperiod of the planning period in order to prevent unrealistic concentrations of investment in short periods of time. Limitations to the emissions of pollutants $\left(\mathrm{SO}_{2}, \mathrm{NO}_{x}\right.$ and $\left.\mathrm{CO}_{2}\right)$ have also been considered.

The MOMILP model aims at providing decision support to DMs and planners to select the types and total capacity of generating units to be installed and the operating levels of the various power units throughout the planning period.

\subsection{Objective functions}

The MOMILP model considers three objective functions, which quantify the total expansion cost, a measure of the environmental impact and the environmental cost (a monetized measure of environmental damages), all to be minimized. The environmental impact objective functions attempt to capture the increasing awareness of environmental externalities resulting from energy generation.

Total expansion cost involves the terms: investment costs $(\$ / \mathrm{MW})$ corresponding to the units to be installed throughout the planning period; operational and maintenance charges $(\$ / \mathrm{MW} \mathrm{h})$ of existing and new units.

$$
\min f_{1}=\sum_{j=1}^{J}\left\{\sum_{r=1}^{j}\left[\left(\frac{g_{r}}{100}\right) \sum_{i=1}^{I} c_{i} x_{i}^{r}\right]+K\left(\sum_{s=1}^{S} s \sum_{i=1}^{I} b_{i} z_{i s}^{j}+\sum_{s=1}^{S} s \sum_{a=1}^{A} b_{a} z_{a s}^{j}\right)\right\}
$$

$j$ is the index of subperiod within the planning period $(j=1, \ldots, J), s$ is the index of interval within a subperiod $(s=1, \ldots, S), i$ is the index of unit type considered for additions $(i=1, \ldots, I), a$ is the index of a unit type existing at the beginning of the planning period 
$(a=1, \ldots, A), x_{i}^{r}$ is the decision variable representing the total power output (MW) of a unit of type $i$ installed in subperiod $r, z_{i s}^{j}$ is the variable in the improved $z$-substitute method (MW) corresponding to a group of type $i$ in interval $s$ of subperiod $j, z_{a s}^{j}$ is the variable in the improved $z$ substitute method (MW) corresponding to a group of type $a$ in interval $s$ of subperiod $j, c_{i}$ is the investment cost $(\$ / \mathrm{MW})$ associated with a group of type $i, b_{i}$ is the operational and maintenance charges $(\$ / \mathrm{MW} \mathrm{h})$ of a group of type $i, b_{a}$ is the operational and maintenance charges $(\$ /$ MW h) of a group of type $a, g_{r}$ is the sum of the annualized capital charges (in percentage of initial investment) at subperiod $r$ of the planning period, $K$ is the number of hours within an interval.

The costs of the equivalent DSM generating unit involve investment costs $(\$ / \mathrm{MW})$, related to the needed hardware and its installation, and operating costs $(\$ / M W h)$. This permits including the DSM unit in the cost objective function in the same manner as the other supply-side options. Investment costs include equipment, installation, removal and initial cost of signing customers. Operating costs include operating and maintenance charges, incentives to consumers and the costs related to the loss of income due to a decrease of $\mathrm{kW} \mathrm{h}$ sales.

The environmental impact associated with the generating capacity to be installed throughout the planning period is quantified by the objective function:

$$
\min f_{2}=\sum_{i=1}^{I} v_{i} \sum_{j=1}^{J} x_{i}^{j}
$$

where $v_{i}$ is a (dimensionless) environmental impact coefficient computed by using a weighted average of the impact evaluation criteria associated with the installation of generating facilities: land use, large accidents and effects on ecosystems.

The environmental cost objective function minimizes the monetized environmental externalities associated with the energy output from each type of power plant:

$$
\min f_{3}=\sum_{j=1}^{J} K\left(\sum_{s=1}^{S} s \sum_{i=1}^{I} e_{i} z_{i s}^{j}+\sum_{s=1}^{S} s \sum_{a=1}^{A} e_{a} z_{a s}^{j}\right)
$$

where $e_{i}$ and $e_{a}$ are the environmental costs $(\$ / \mathrm{MW} \mathrm{h})$ associated with the energy output of groups of type $i$ considered for additions and existing units of type $a$, respectively. The coefficients $e_{i}$ and $e_{a}$ are computed by using a weighted average of impact evaluation criteria associated with energy output: emissions/public health, water use and occupational accidents.

\subsection{Constraints}

Reliability requirements are coped for by observing the principle according to which power units in operation must satisfy the instantaneous power demand as well as provide a reserve margin, which is defined as a percentage of peak demand throughout the planning period. This leads to two groups of constraints expressed in terms of installed capacities and in terms of 
operating variables:

$$
\begin{aligned}
& \sum_{t=1}^{j} \sum_{i=1}^{I} x_{i}^{t}+\sum_{a=1}^{A} G_{a} \geq P_{1}^{j}+R P_{1}^{j} \quad(j=1, \ldots, J) \\
& \sum_{i=1}^{I} \sum_{s=k}^{S} z_{i s}^{j}+\sum_{a=1}^{A} \sum_{s=k}^{S} z_{a s}^{j} \geq P_{k}^{j} \quad(j=1, \ldots, J, \quad k=1, \ldots, S)
\end{aligned}
$$

$P_{k}^{j}$ is the power demand in interval $k$ of subperiod $j$ (the peak load in subperiod $j$ corresponds to $\left.P_{1}^{j}\right), R$ is the fraction of peak demand considered as an adequate reserve margin for each subperiod $j$ of the planning period, and $G_{a}$ is the installed power of existing units of type $a$.

As far as the operation of generating units is concerned, the power that can be generated by any type of unit cannot exceed its rated capacity affected by an availability factor.

For existing facilities:

$$
\sum_{s=1}^{S} z_{a s}^{j} \leq m_{a} G_{a} \quad(j=1, \ldots, J, \quad a=1, \ldots, A)
$$

in which $m_{a}$ is the availability factor.

For new units:

$$
\sum_{s=1}^{S} z_{i s}^{j} \leq m_{i} \sum_{r=1}^{j} x_{i}^{r} \quad(j=1, \ldots, J, \quad i=1, \ldots, I)
$$

where $m_{i}$ is the availability factor of a unit of type $i$.

An upper bound of a certain percentage of the peak demand is imposed on the operation of the equivalent DSM generating unit ( $i=$ DSM unit), at each subperiod:

$$
\sum_{s=k}^{T} z_{i s}^{j} \leq u P_{k}^{j} \quad(j=1, \ldots, J, \quad k=1, \ldots, T)
$$

in which $T$ is the number of intervals of highest demand values in the LDC of subperiod $j$ when DSM options are allowed (thus acting as a peak avoiding alternative), $u$ is the fraction of required peak demand that is considered to be installed in the DSM unit, at each subperiod $j$ of the planning period. These constraints also avoid the possibility of an unrealistically high penetration of DSM at certain subperiods.

An upper bound $\left(U_{j}\right)$ on the new power generating capacity of all types to be installed at each subperiod $j$ of the planning period is also imposed to account for technical and budgetary 
limitations:

$$
\sum_{i=1}^{I} x_{i}^{j} \leq U_{j} \quad(j=1, \ldots, J)
$$

For each generating unit installed throughout the planning period, its available modular expansion must be considered:

$$
x_{i}^{j}=\sum_{m=1}^{M i} \alpha_{i m} y_{i m}^{j} \quad(i=1, \ldots, I, j=1, \ldots, J)
$$

where $y_{i m}^{j}$ is an integer variable representing the number of capacity module $m$ corresponding to a group of type $i$ (whose rated power is $\alpha_{i m}$ ) installed in subperiod $j$ and $M_{i}$ is the number of modules available corresponding to a group of type $i$.

Upper bounds for sulphur-dioxide $\left(\mathrm{SO}_{2}\right)$, nitrogen-oxide $\left(\mathrm{NO}_{x}\right)$, and carbon-dioxide $\left(\mathrm{CO}_{2}\right)$ emissions are imposed for taking into account regulations on pollutant emissions resulting from electricity production:

$$
\begin{aligned}
& 10^{-6} K \sum_{a=1}^{A} h_{a} \sum_{s=1}^{S} s z_{a s}^{j} \leq S H_{a}^{j} \quad(j=1, \ldots, J) \\
& 10^{-6} K \sum_{a=1}^{A} l_{a} \sum_{s=1}^{S} s z_{a s}^{j} \leq S L_{a}^{j} \quad(j=1, \ldots, J) \\
& 10^{-6} K \sum_{a=1}^{A} t_{a} \sum_{s=1}^{S} s z_{a s}^{j} \leq S T_{a}^{j} \quad(j=1, \ldots, J) \\
& 10^{-6} K \sum_{i=1}^{I} h_{i} \sum_{s=1}^{S} s z_{i s}^{j} \leq S H_{i}^{j} \quad(j=1, \ldots, J) \\
& 10^{-6} K \sum_{i=1}^{I} t_{i} \sum_{s=1}^{S} s z_{i s}^{j} \leq S T_{i}^{j} \quad(j=1, \ldots, J) \\
& 10^{-6} K \sum_{i=1}^{I} l_{i} \sum_{s=1}^{S} S z_{i s}^{j} \leq S L_{i}^{j} \quad(j=1, \ldots, J)
\end{aligned}
$$

where $h_{a} / l_{a} / t_{a}\left(h_{i} / l_{i} / t_{i}\right)$ are the emissions levels $(\mathrm{g} / \mathrm{kW} \mathrm{h})$ of $\mathrm{SO}_{2} / \mathrm{NO}_{x} / \mathrm{CO}_{2}$ for a unit of type $a$ (i), respectively. $H_{a}^{j} / L_{a}^{j} / T_{a}^{j}\left(H_{i}^{j} / L_{i}^{j} / T_{i}^{j}\right)$ are the upper bounds (kton/subperiod) on emissions of $\mathrm{SO}_{2} / \mathrm{NO}_{x} / \mathrm{CO}_{2}$ for a unit of type $a(i)$ in subperiod $j$, respectively.

\section{An illustrative example}

This section is aimed at illustrating how the interactive approach described in Section 3 can be used to provide decision support for selecting a satisfactory compromise solution based on 
the MOMILP power generation expansion model with a data realization. The data used to construct the model coefficients (Tables 1-7) have been gathered from several sources with a strong concern to be as much as possible in agreement with real values (and paying attention to the Portuguese case), although not corresponding to an actual case study. The planning period consists of three subperiods, each one divided into 12 semesters (intervals). The MOMILP model considers three objective functions, 93 constraints, 222 continuous decision variables and 18 integer decision variables. Oil- and coal-fired generating units are in operation at the beginning

Table 1

Generating system existing at the beginning of the planning period

\begin{tabular}{lllll}
\hline Type & $\begin{array}{l}\text { Total power } \\
(\mathrm{MW})\end{array}$ & $\begin{array}{l}\text { Operational costs } \\
(\$ / \mathrm{MW} \mathrm{h})\end{array}$ & $\begin{array}{l}\text { Environmental costs } \\
(\$ / \mathrm{MW} \mathrm{h})\end{array}$ & Availability factor \\
\hline Coal & 1880 & 20.0 & 105.70 & 0.75 \\
Oil & 2400 & 26.6 & 93.39 & 0.80 \\
\hline
\end{tabular}

Table 2

Generating units considered for additions

\begin{tabular}{llllll}
\hline Type & $\begin{array}{l}\text { Investment costs } \\
(\$ / \mathrm{KW})\end{array}$ & $\begin{array}{l}\text { Operational costs } \\
(\$ / \mathrm{MW} \mathrm{h})\end{array}$ & $\begin{array}{l}\text { Environmental } \\
\text { impact }\end{array}$ & $\begin{array}{l}\text { Environmental } \\
\text { costs }(\$ / \mathrm{MW} \mathrm{h})\end{array}$ & $\begin{array}{l}\text { Availability } \\
\text { factor }\end{array}$ \\
\hline Coal & 950 & 16.2 & 4.33 & 105.70 & 0.80 \\
Gas (simple) & 195 & 29.6 & 2.67 & 39.43 & 0.90 \\
Gas (combined) & 375 & 20.7 & 2.56 & 25.71 & 0.70 \\
DSM options & 211 & 75.1 & 0 & 0 & 0.95 \\
\hline
\end{tabular}

Table 3

Peak load duration curves (MW)

\begin{tabular}{|c|c|c|c|c|c|c|c|c|c|c|c|c|}
\hline & 1 & 2 & 3 & 4 & 5 & 6 & 7 & 8 & 9 & 10 & 11 & 12 \\
\hline 1 & 3791 & 3727 & 3663 & 3600 & 3538 & 3476 & 3415 & 3355 & 3295 & 3236 & 3178 & 3117 \\
\hline 2 & 4613 & 4541 & 4470 & 4399 & 4329 & 4259 & 4190 & 4122 & 4054 & 3988 & 3922 & 3860 \\
\hline 3 & 5526 & 5447 & 5368 & 5290 & 5212 & 5135 & 5058 & 4982 & 4907 & 4833 & 4759 & 4695 \\
\hline
\end{tabular}

Table 4

Coefficients and upper bounds for pollutant emissions

\begin{tabular}{|c|c|c|c|c|c|c|c|c|}
\hline \multirow[t]{2}{*}{ Pollutant } & \multicolumn{5}{|c|}{ Coefficient emission by unit type $(\mathrm{g} / \mathrm{KW} \mathrm{h})$} & \multicolumn{3}{|c|}{$\begin{array}{l}\text { Upper bound at each subperiod (kton/ } \\
\text { year) }\end{array}$} \\
\hline & Coal & Oil & $\begin{array}{l}\text { Coal } \\
\text { (new) }\end{array}$ & $\begin{array}{l}\text { Gas } \\
\text { (simple) }\end{array}$ & $\begin{array}{l}\text { Gas } \\
\text { (combined) }\end{array}$ & 1 & 2 & 3 \\
\hline $\mathrm{SO}_{2}$ & 5.84 & 14.30 & 4.00 & 0 & 0 & 226 & 214 & 185 \\
\hline $\mathrm{NO}_{x}$ & 2.93 & 2.49 & 2.20 & 0.028 & 0.018 & 46 & 44 & 42 \\
\hline $\mathrm{CO}_{2}$ & 890 & 753 & 830 & 448 & 285 & 15,000 & 14,000 & 12,000 \\
\hline
\end{tabular}


Table 5

Environmental impact coefficients

\begin{tabular}{lllll}
\hline Criteria & Land use & Large accidents & Effect on ecosystems & Weighted average \\
\hline Weight & 1 & 3 & 5 & \\
Coal & 5 & 3 & 5 & 4.33 \\
Oil & 3 & 5 & 4 & 4.22 \\
Gas (simple) & 3 & 2 & 3 & 2.67 \\
Gas (combined) & 4 & 3 & 2 & 2.56 \\
DSM options & 0 & 0 & 0 & 0 \\
\hline
\end{tabular}

Table 6

Environmental impact cost coefficients

\begin{tabular}{lccc}
\hline Criteria & $\begin{array}{l}\text { Emissions/public health } \\
(\$ / \mathrm{MW} \mathrm{h})\end{array}$ & $\begin{array}{l}\text { Occupational accidents } \\
(\$ / \mathrm{MW} \mathrm{h})\end{array}$ & $\begin{array}{l}\text { Weighted average } \\
(\$ / \mathrm{MW} \mathrm{h})\end{array}$ \\
\hline Weight & 5 & 2 & \\
Coal & 113.9 & 85.2 & 105.70 \\
Oil & 112.5 & 45.6 & 93.39 \\
Gas (simple) & 38.0 & 43.0 & 39.43 \\
Gas (combined) & 20.0 & 40.0 & 25.71 \\
DSM options & 0 & 0 & 0 \\
\hline
\end{tabular}

Table 7

Rated power of capacity modules (MW)

\begin{tabular}{llll}
\hline$\alpha_{i m}$ & 1 & 2 & 3 \\
\hline Coal & 300 & 450 & 700 \\
Gas (simple) & 160 & 400 & \\
Gas (combined) & 330 & & \\
\hline
\end{tabular}

of the planning period. The generation technologies considered for additions are coal and natural gas (both simple and combined cycle) besides DSM options.

The reserve margin is $R=20 \%$ of peak load for each interval of each subperiod. The upper bound on the new capacity (except DSM options) which is allowed to be installed in each subperiod is $1750 \mathrm{MW}$.

An upper bound of $7.5 \%$ of the peak load is imposed on the capacity of DSM options at each subperiod. The DSM unit can be in operation in the first four intervals of each subperiod only.

The number of hours within an interval $(K)$ is 4380 .

The nondominated solutions (to the MOLP) which optimize each objective function individually are computed (solutions $\mathrm{C} 1, \mathrm{C} 2$ and $\mathrm{C} 3$ in Table 8). The components of the (continuous) ideal solution, which is the initial reference point, are displayed in bold.

The nondominated solution which minimizes the Tchebycheff distance to the ideal solution is then computed (solution C4 in Table 8).

Let us suppose that, regarding solution $\mathrm{C} 4$, the $\mathrm{DM}$ is willing to relax $f_{3}$ by $0.2 \times 10^{10}$ in order to improve the other functions. The following constraints are added to the problem for- 
Table 8

Continuous nondominated solutions

\begin{tabular}{lcrc}
\hline Solution & $f_{1}\left(10^{10}\right)$ & $f_{2}\left(10^{5}\right)$ & $f_{3}\left(10^{10}\right)$ \\
\hline C1 & 1.9642 & 10.7630 & 4.2971 \\
C2 & 2.1328 & $\mathbf{9 . 5 3 3 2}$ & 4.0347 \\
C3 & 2.2001 & 14.0175 & $\mathbf{3 . 6 0 1 0}$ \\
C4 & 2.1655 & 11.5466 & 3.8023 \\
C5 & 2.0605 & 11.4967 & 4.0023 \\
\hline
\end{tabular}

mulation:

$$
\begin{aligned}
& f_{1} \leq 2.1655 \times 10^{10} \\
& f_{2} \leq 11.5466 \times 10^{5} \\
& f_{3} \leq 3.8023 \times 10^{10}+0.2 \times 10^{10}
\end{aligned}
$$

The new (continuous) nondominated solution obtained in the reduced feasible region is solution C5 in Table 8.

If the DM considers this solution a satisfactory one, then it is taken as the reference point and the nondominated solution (to the MOMILP) which minimizes the Tchebycheff distance to it is computed. The obtained solution is I1, whose objective function values are displayed in Table 9 and the corresponding generation additions are presented in Table 10.

Let us suppose the DM wants to improve the value of $f_{1}$ in this solution. To do this, he/she can shift the $f_{1}$ component of the reference point (thus implicitly specifying a new reference point he/she would like to attain), for instance from 2.0605 to 2.0105 . Therefore, a new continuous nondominated solution (to the MOLP) which minimizes the Tchebycheff distance to this reference point is computed (see flowchart in Fig. 2). If this solution is considered satisfactory by the DM, then it is taken as the new reference point. The integer nondominated solution closest to this new reference point, according to the Tchebycheff metric, is solution I2 (see Tables 9 and 10). The additional (soft) constraints imposed above to reduce the feasible region could also be revised in later interactions with the DM.

DSM options are present in almost all computed solutions (except near the optimum of the cost objective function), although its available capacity, as defined by the corresponding constraints, is never fully used. This happens, even though the coefficients related to DSM options (modelled as a generating technology) are 0 in $f_{2}$ and $f_{3}$, because of two cumulative effects: the need to install generating capacity from other technologies which is necessary to satisfy the

Table 9

Integer nondominated solutions

\begin{tabular}{llll}
\hline Solution & $f_{1}\left(10^{10}\right)$ & $f_{2}\left(10^{5}\right)$ & $f_{3}\left(10^{10}\right)$ \\
\hline I1 & 2.0751 & 10.5632 & 4.0169 \\
I2 & 2.0488 & 10.7920 & 4.0406 \\
\hline
\end{tabular}


Table 10

Addition of generation capacity for the nondominated integer solutions $(\mathrm{SC}=$ simple cycle; $\mathrm{CC}=$ combined cycle; the index refers to subperiods)

\begin{tabular}{llllllllll}
\hline Solution & Gas & Gas & DSM1 & Gas & Gas & DSM2 & Gas & Gas & DSM3 \\
& SC1 & CC1 & & SC2 & CC2 & & SC3 & CC3 & \\
\hline I1 & 960 & 730 & 127.5 & 640 & 1060 & & 640 & & \\
I2 & 960 & 730 & 127.5 & & 1650 & & 800 & & \\
\hline
\end{tabular}

demand in intervals $s>4$, when DSM options are not permitted, and the modular nature of the capacity of generating units installed throughout the planning period.

Other search directions could have been pursued in the operational framework of this interactive approach in order to exploit other regions of the nondominated solution set. Although in this example only information concerning the values of the objective functions and the generation additions has been presented, the DM is offered all the solution attributes (including pollutant emissions, etc.) which can be used to refine and guide the search process as well as to identify a solution as a satisfactory compromise plan.

\section{Conclusions}

An MOMILP model has been presented to provide decision support in the evaluation of power generation capacity expansion policies. The model takes explicitly into account multiple evaluation aspects, DSM issues, and the modularity of expansion possibilities. DSM is modelled as an equivalent of a generating group, similar to the generating alternatives from the supply side with some operational restrictions. The objective functions are the total expansion cost, the environmental impact associated with the installed power capacity and the environmental impact associated with the energy output.

Nondominated solutions to the MOMILP model are computed by means of an interactive algorithm based on a reference direction approach, which is not too demanding regarding the computational burden and the information required from the decision maker.

This model not just accounts for investment and operation costs associated with DSM but also considers the costs of lost revenues due to DSM actions. Therefore, the factors penalizing DSM actions are embedded in the model.

As far as the transformations currently underway in the energy market are concerned, the model can also be applied, besides the case where the utility is still vertically integrated, in a context in which private investment is invited to fulfil capacity quotas previously defined in a strategic development plan.

Studies dealing with the sensitivity and robustness analyses of nondominated solutions, regarding changes in both the input coefficients and the DM's preferences, are currently underway.

\section{Acknowledgements}

This work was supported in part by the Foundation for Science and Technology (FCT) under Grant POCTI/ESE/38422/2001. 


\section{References}

[1] Beglari F, Laughton M. Model building with particular reference to power system planning: the improved $z$ substitute method. Energy Modelling 1974:56-65.

[2] Benayoun R, de Montgolfier J, Tergny J, Larichev O. Linear programming with multiple objective functions: step method (STEM). Math Program 1971;1(3):366-75.

[3] Clímaco J, Antunes CH, Martins AG, Almeida AT. A multiple objective linear programming model for power generation expansion planning. Int J Energy Res 1995;19:419-32.

[4] Gellings CW, Smith WM. Integrating demand-side management into utility planning. Proc IEEE 1989;77(6):908-18.

[5] Hobbs B, Meier P. Energy decisions and the environment - a guide to the use of multicriteria methods. Boston: Kluwer Academic Publishers; 2000.

[6] Martins AG, Coelho D, Antunes CH, Clímaco J. A multiple objective linear programming approach to power generation planning with demand-side management. Int Trans Oper Res 1996;3(3/4):305-17.

[7] Mavrotas G, Diakoulaki D, Papayannakis L. An energy planning approach based on mixed 0-1 multiple objective linear programming. Int Trans Oper Res 1999;6:231-44.

[8] Mills D, Vlacic L, Lowe I. Improving electricity planning - use of a multicriteria decision making model. Int Trans Oper Res 1996;3(3/4):293-304.

[9] Narula SC, Vassilev V. An interactive algorithm for solving multiple objective integer linear programming problems. Eur J Oper Res 1994;79:443-50.

[10] Steuer R. Multiple criteria optimization: theory, computation and application. New York: Wiley; 1986. 